\title{
Superior SOM Neural Network based Minute Significant Watermark Generator and Detector System
}

\author{
N. Chenthalir Indra \\ Assistant Professor \\ S.T.Hindu College \\ Tamil Nadu, India.
}

\author{
Dr. E. Ramaraj \\ Technology Advisor \\ Madurai Kamaraj University \\ Tamil Nadu, India
}

\begin{abstract}
This paper suggests the Superior SOM (SSOM) based Minute Significant Watermark Generator \& Detector (MSWG\&D) system. RGB features of the host image are trained in different SSOM networks. Subsequent to SSOM training process, microscopic significant values are synthesized from host image and self-possessed as watermark values. Then these values are embedded into the high frequency sub band of Discrete Wavelet Transform (DWT). The Quality of invisible watermarking is proved by evaluating PSNR \& Jaccard Similarity Ratio values between original and watermarked image. MSWG\&D system is robust to JPEG compression and noise attacks. The experimental results prove that the strength of proposed watermarking system is 'one more landmark' in the watermarking techniques.
\end{abstract}

\section{General Terms}

Watermarking, Self Organizing Neural Network, Discrete Wavelet Transform.

\section{Keywords}

Superior Self Organizing Maps, Minute significant watermark,

\section{INTRODUCTION}

Modern visualization techniques warehouse digital image data. Image data handling through network uses digital watermarking security. Digital image watermarking is a technique which embeds additional information called digital signature or watermark into the digital content in order to secure it [11]. Satisfactory digital watermarking must meet the following requirements:

- Robustness: the watermark must be difficult to delete and should be resistant to standard image processing operations such as filtering, cropping, loose compression, etc.

- Imperceptibility: the watermark embedded in the image should not be caused obvious visual degradation of images.

- Security: the attacker should not be able to detect the embedded watermark by using common statistical analysis or correlative attacks [4].

This paper proposes an alternative Neural Network based watermarking technique. Common contribution of neural networks in watermarking was embedding digital signal into the host image. The proposed system successfully generates watermark digital signal from the host image itself by using Self Organized Maps (SOM) Network concept. Since digital signature is created from host image the watermark value is unique for every image. The illegal claim or attacks can be easily identified for the trust full image communication process by detection system. Section 2 gives details about the system background for the planned watermarking framework. The proposed system is described in the Section 3. The planned watermarking technique is analyzed by applying the test on 128 x 128 size color image. Also the efficiency of the proposed system is proved by comparing the results with traditional SOM. Section 5 concludes the strength of proposed watermarking system as one more landmark in the watermarking techniques.

\section{REQUIRED BACKGROUND}

The paper [1] exhibits that, a number of techniques have been proposed for digital image and digital video watermarking by training neural networks. The neural networks that are used for watermarking include Back propagation Network (BPN), Counter Propagation Network (CPN), Full Counter Propagation Network, Cellular Neural Network (CNN), Radial Basis Function Neural Network (RBF) and Hopfield Neural Network.

C.-Y. Chang et al. [5] used a full counter-propagation neural network (FCNN) for copyright protection where the ownership information was embedded and detected by a specific FCNN. A BPN (Back Propagation Network) model is used to learn the relationship between the watermark and the watermarked image [8]. Zhang [7] proposed a blind watermarking algorithm using Hopfield neural network and then analyzed the watermarking capacity based on the neural network.

The existing techniques used neural networks for embedding and finding strength of watermarking. And moreover 'Self Organizing Maps' was not directly used for watermark generation in the early papers. This paper proposes 'Superior Self Organizing Maps' (SSOM) for watermark value generation and detection process. No separate watermark values are used. 
Watermark value is generated from the host image itself. Host image is a vital image to be transferred. Watermark value is embedded in the same image as self signature value.

The potentialities of the conventional SOM technique have been extensively explored in different research areas for more than two decades [6]. As in any clustering logics SOM is much influenced by its distance calculation step. Conventional SOM uses Euclidean distance or the dot product as its winning neuron selector to train the weight vector nodes. The previous paper [2] proved that the Maximum Value distance based SOM attains its maximum knowledge about an image is up to $99 \%$ with minimum epochs. Another previous analysis established that the measures Manhattan and Lee bring $99.5 \%$ support to SOM in image learning process [3]. The above said improvised SOM is better than the conventional SOM. Hence the improvised SOM neural network is mentioned as Superior Self Organizing Maps (SSOM)

\subsection{Superior SOM Learning Algorithm}

Step0: Initialize weights with random method or by having previous knowledge of Pattern distribution. Set Topological neighborhood parameters. Set learning rate parameter

Step1: While stopping condition is false, do steps $2-8$

Step2: For each input vector $\mathbf{x}$, do steps $3-5$

Step3: For each $\mathbf{j}$, compute $d(j)$ by using any one of the following distance measures Eqns.(1) or (2).

a) Manhattan distance :

$$
d(x, y)=\sum_{i=1}^{M}\left|x_{i}-y_{i}\right|
$$

b) Lee distance :

$$
d_{m}(x, y)=\sum_{i=1}^{M}\left\{\left|x_{i}-y_{i}\right|, q-\left|x_{i}-y_{i}\right|\right\}
$$

Step4: Find index $\mathbf{J}$ such that $\mathbf{d}(\mathbf{j})$ is a minimum.

Step5: For units $\mathbf{j}$ within a specified neighborhood of $\mathbf{J}$ and for all $\mathbf{i}$

$\mathrm{W}_{\mathrm{ij}}($ new $)=\mathrm{W}_{\mathrm{ij}}($ old $)+\alpha\left[\mathrm{X}_{\mathrm{i}}-\mathrm{W}_{\mathrm{ij}}(\right.$ old $\left.)\right]$

Step6: Update learning rate

Step7: Reduce radius of topological at specified times

Step8: Test stopping condition.

The Learning Rate is a slowly decreasing function of time. The radius of the neighborhood around a cluster unit also decreases as the clustering process progresses. The updated weight network is well equipped with host image neural structure. SSOM exactly imitates human neural learning logic. Hence trivial imbalanced values can be identified through the analysis. These insignificant map elements in SSOM network is determined and used as watermark values.

\subsection{Discrete Wavelet Transform}

The first DWT was invented by the Hungarian mathematician Alfred Haar. For an input represented by a list of $2^{n}$ numbers, the Haar wavelet transform may be considered to simply pair up input values, storing the difference and passing the sum. This process is repeated recursively, pairing up the sums to provide the next scale: finally resulting in $2^{n}-1$ differences and one final sum.

The proposed algorithm is a frequency domain watermarking scheme and works by modifying the DWT coefficients. The wavelet transform decomposes input image into four components namely LL, HL, LH and HH. The lowest resolution level LL consists of the approximation part of the original image. The remaining three resolution levels consist of the detail parts and give the vertical high (LH), horizontal high (HL) and high $(\mathrm{HH})$ frequencies.

In the proposed technique, embedding and extraction of watermark takes place in the high frequency component. For a one level decomposition, the discrete two-dimensional wavelet transform of the image function $\mathrm{f}(\mathrm{x}, \mathrm{y})$ is found in [9] [10]. DWT transform based watermarking scheme is robust against many common image attacks. The analysis results proved robustness very well.

\section{PROPOSED WATERMARKING SYSTEM}

Digital watermarking is the process of embedding information into a digital signal in a way that is difficult to remove. Using SSOM is safer than any other neural algorithms because it is an unsupervised learning process and minimal variation in image will be reflected in the watermark values. The training process is public but the watermark value generation is based on the weight initialization, learning rate fixation, and training epoch value and learning ability of network. Hence unauthorized users can not generate the watermark values. RGB features of the host image are trained in different SSOM networks. Subsequent to SSOM training process, microscopic significant values are synthesized from host image and self-possessed as watermark values. Then these values are embedded into the high frequency sub band of Discrete Wavelet Transform (DWT). 


\subsection{Minute Significant Watermark Generation \& Embedding \\ A) Preprocessing the image:}

1) Collect the input images. Select the host image for watermarking. Read its RGB values (3-D) as $X(x, y, z)$

2) Extract its Red, Green and Blue colour attributes in separate 2-D spaces $\operatorname{Xr}(x, y), X g(x, y)$ and $X b(x, y)$ respectively.

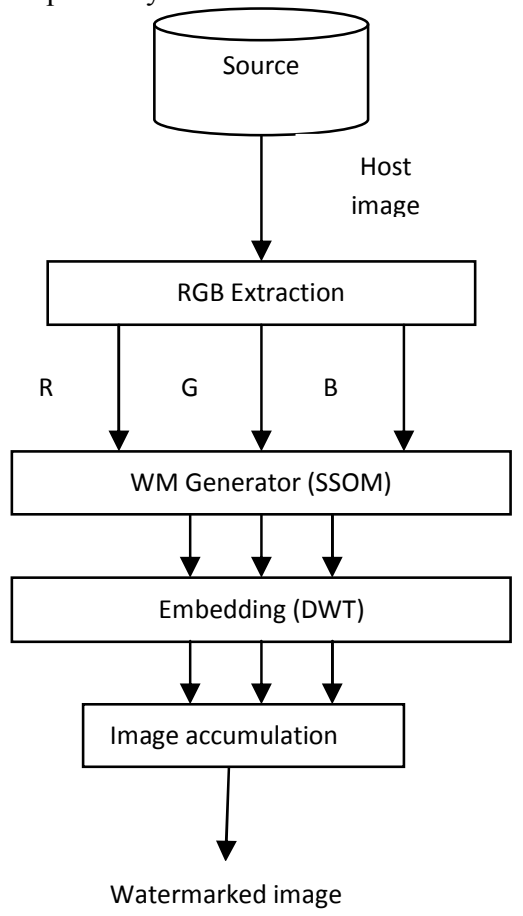

Figure 3.1. Minute Significant Watermark Generation and embedding process

B) Water mark Generation

1) Set the SSOM network with three layers. (Input, three hidden and output with two dimensional space matrix).

2) Initialize its weight vectors with integer numbers by using any existing auto random number generator function. Red, green and blue related SOM weight vectors are $W r(x, y), W g(x, y)$ and $W b(x, y)$ respectively (random numbers between 0 and 255 integer values).

3) Initialize learning rate $\alpha$ as 0.8 (this threshold value may vary between 0.5 and 1.0 ).

4) Red, Green and Blue feature 2-D matrix values are supplied to the input layer of SOM network. Train the network by using the feasible measures given in the SSOM.

5) Trained RGB attributes are obtained in the output layer.
6) Find the difference between input values and trained values of each colour. The resultant values are accepted as digital watermark values. Thus the first phase obtains three sets of digital watermarks.

C) Embedding Watermark:

1) Activate 1-level DWT to original image's red vectors.

2) The red attribute watermark is embedded in the high frequency component $\mathrm{HH}$ of DWT.

3) Execute inverse wavelet transform to obtain the watermarked red features.

4) Repeat the above three steps for other two green and blue colours too.

D) Watermarked image accumulation:

1) Collect watermarked 2-D space values of each colour.

2) Combine them together into 3-D space data type.

3) Store the resultant 3-D image values by using .jpg format. (compressed watermarked host image is ready for transmission).

Fig. 3.1. shows Minute Significant Watermark Generation and embedding process

\subsection{Minute Significant Watermark Detection and Separation process}

1) Projected watermarking proposal is capable of mine watermark information in the absence of the original image or secret key. Hence it is unsighted watermarking.

2) Regenerate watermark from the transferred image by using SSOM neural logic as mentioned in the generation algorithm.

3) Trigger one level DWT to the destination image and take away the embedded watermark from the $\mathrm{HH}$ sub band.

Fig. 3.1 (b) shows Minute Significant watermark detection and separation process. The resultant image must be matched with the original if it is not disturbed during transmission. Quality of transferred watermarked image is analyzed by using PSNR measure given in equation (3).

$$
P S N R=10 \log _{10}\left(\frac{255^{2}}{M S E}\right)
$$




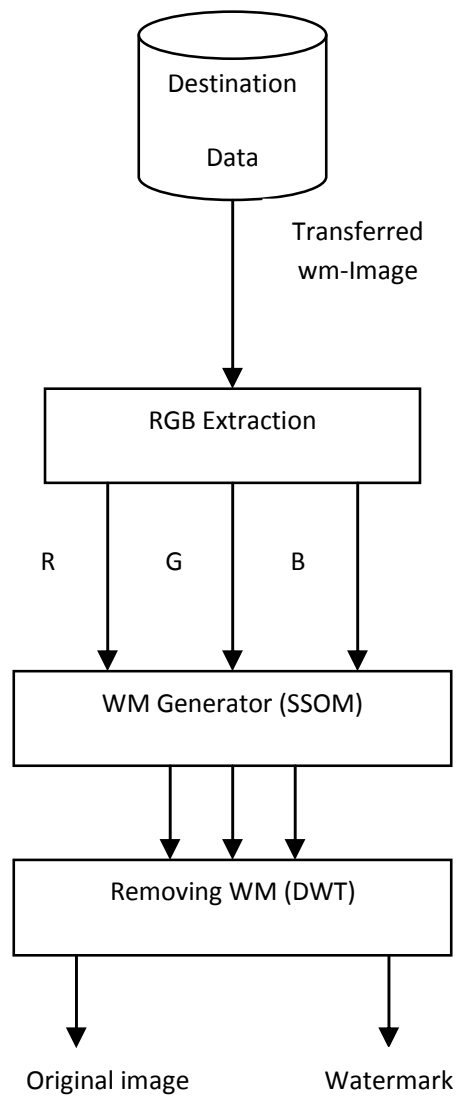

Figure 3.2. Minute Significant Watermark Detection and separation process.

Where, MSE is Mean Squared Error between original image and watermarked image. Similarity ratio between the host image and watermarked image is calculated by using Jaccard similarity measure given in the equation (4). The similarity ratio (SR) is 1 for the exactly same images and 0 for the entirely different images. This paper accepts the range from 0.8 to 1 as the best validity of similarity ratio.

Jaccard coefficient:

$\operatorname{sim}\left(x_{i}, w_{j}\right)=\frac{\sum_{h=1}^{k} x_{i h} w_{j h}}{\sum_{h=1}^{k} x_{i h}^{2}+\sum_{h=1}^{k} w_{j h}^{2}-\sum_{h=1}^{k} x_{i h} w_{j h}}$

\section{ANALYSIS RESULTS}

The host image was selected and given as input to the MSWG system. The RGB feature values are extracted and three sets of 2-D matrix values are constructed according to the image pattern. These RGB 2-D plane are trained by SSOM algorithm with its authenticated initial settings. The trained network weight vectors are compared with host image color pixel values. Minute significant values are identified as digital watermark. The digital watermark is embedded by using single level DWT.

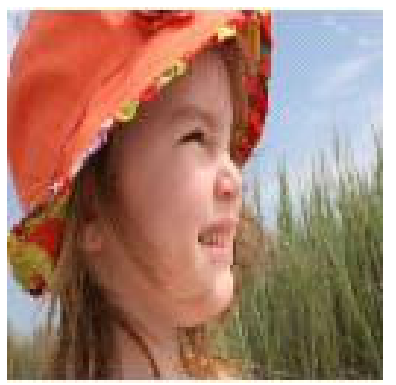

(a)

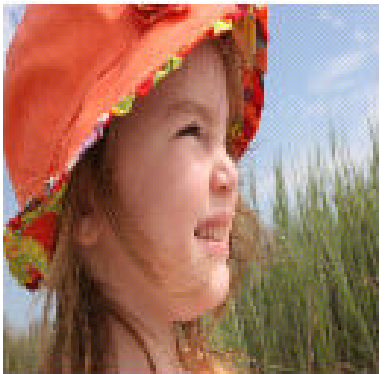

(b)

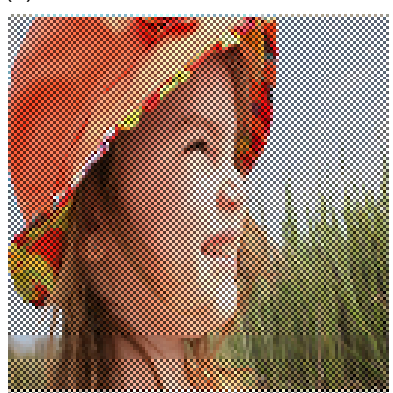

(c)
Figure 4.1 (a)Host image (b)SSOM_Watermarked image (c) SOM_watermarked image

No techniques can guess these water mark values because the SOM is unsupervised learner and moreover the experiment uses random numbers to initialize the weight vector of hidden layer. Results were analyzed with two modes one by Conventional SOM and other with proposed Superior SOM. Fig. 1.1 is evidence for the watermark embedded image which is visually degraded with traditional SOM. But with Superior SOM the watermarked image is impeccable. The robustness of watermark is further tested by introducing various mild attacks.

No visible change in image was found in the proposed watermarking scheme. Even though watermark casting process is in open, the intruders or unofficial users cannot identify or remove the watermark from the watermarked image with its threshold measure, since the SSOM is an unsupervised neural network. SSOM is the improvised version of traditional SOM neural network. It trains the host image at its maximum level. Hence the watermark values are very small digital value, embedding those values will not change the clarity of image.

\subsection{Watermarking \& Transmission level test}

The watermarked image is compared with original host image. As per theory $25 \mathrm{~dB}$ PSNR value is acceptable. Above $40 \mathrm{~dB}$ PSNR is proposed by the MSWG\&D system because the main aim of the scheme is to use the images for copyright applications. PSNR with less than $40 \mathrm{~dB}$ are good but not relevant for authentication. The reliability of watermarking is verified by means of JPG compression and marked in table 4.1.1. The similarity ratio is very high and noticeably no change was identified. Even the compression process made no remarkable modifications in the watermarked image. Traditional 
SOM watermarking yields visually degraded image and the PSNR range is not up to the decided level in the suggested system. SR ratio is very poor when compared with Superior SOM based watermarking. SSOM based Watermarking assures 0.99 of similarity ratio where as the Conventional SOM based watermarking SR value is very low.

\begin{tabular}{|l|l|l|l|l|l|}
\hline \multicolumn{2}{|c|}{ Conventional SOM } & \multicolumn{3}{|c|}{ Superior SOM } \\
\hline \multirow{2}{*}{ Image } & \multicolumn{2}{|l|}{ Quality Ratio } & \multirow{2}{*}{ Image } & \multicolumn{2}{l|}{ Quality Ratio } \\
\cline { 2 - 3 } \cline { 5 - 6 } & PSNR & SR & PSNR & SR \\
\hline $\begin{array}{l}\text { Water } \\
\text { marked } \\
\text { image }\end{array}$ & 33 & 0.69 & $\begin{array}{l}\text { Water } \\
\text { marked } \\
\text { image }\end{array}$ & 44.2 & 0.99 \\
\hline $\begin{array}{l}\text { Compr } \\
\text {-essed }\end{array}$ & 33.4 & 0.63 & $\begin{array}{l}\text { Compr } \\
\text {-essed }\end{array}$ & 45.4 & 0.99 \\
\hline
\end{tabular}

Table 4.1.1. Reliability of MSWG watermarking

The noises were introduced with its minimum level. Negligible level noise also decreases the PSNR value. The proposed scheme will deny the image as an unreliable image. Watermarked image with various noise are exhibited in the figure 4.1.2. Watermark value is regenerated by using MSWD system from destination data. In the destination side noisy data are also included for reliability check.

\begin{tabular}{|c|c|c|c|}
\hline \multicolumn{2}{|c|}{ Conventional SOM } & \multicolumn{2}{c|}{ Superior SOM } \\
\hline $\begin{array}{c}\text { Noise } \\
\text { applied }\end{array}$ & $\begin{array}{c}\text { Watermarked } \\
\text { image PSNR } \\
\text { after noise } \\
\text { attack }\end{array}$ & $\begin{array}{c}\text { Noise } \\
\text { applied }\end{array}$ & $\begin{array}{c}\text { Watermarked } \\
\text { image PSNR } \\
\text { after noise } \\
\text { attack }\end{array}$ \\
\hline No attack & 33 & No attack & 37.8 \\
\hline Gaussian & 31.3 & Gaussian & 37.8 \\
\hline Poisson & 32.4 & Poisson & 38.4 \\
\hline $\begin{array}{c}\text { Salt \& } \\
\text { Pepper }\end{array}$ & 30.5 & $\begin{array}{c}\text { Salt \& } \\
\text { Pepper }\end{array}$ & 39.2 \\
\hline Speckle & 31.2 & Speckle & 36.2 \\
\hline
\end{tabular}

Table 4.1.2 Image PSNR after Noise attack

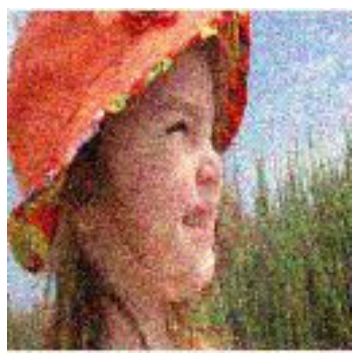

(a)

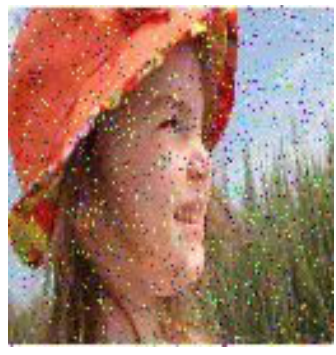

(c)

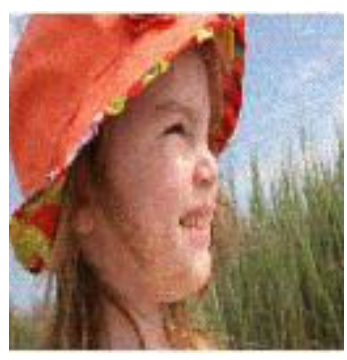

(b)

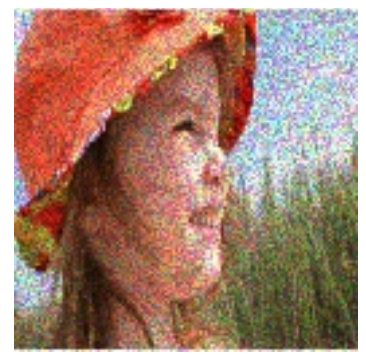

(d)
Figure 4.1.2. Noisy watermarked images (a) Gaussian noise (b) Poissian noise (c) Salt \& Pepper noise (d) Speckle noise

\subsection{Watermark detection level test}

The watermarked signal was regenerated from the receiver side watermarked image and compared with the original watermark signal values to evaluate the robustness of the watermark. PSNR between embedded and detected watermark are measured and noted down in the table 4.2.1. The test was done with both SSOM and SOM networks to prove again the efficiency of SSOM. The PSNR value of detected and regenerated watermark is of higher with proposed SSOM watermarking more than $50 \mathrm{~dB}$. The digital watermark defends against the minimal attacks.

The $100 \%$ original host image can be composed by removing the watermark from the Watermarked image without any attack. Watermark detection and elimination can be done with the Minute Significant Watermark Detection and separation process. With the traditional SOM attacks on the watermarked image degrade the watermarks quality ratio but with SSOM it is maintained with $50 \mathrm{~dB}$.

From the Table 4.2.1 it is proved that the watermark has higher tolerance and it can be recover safely with reliable range of threshold set in this scheme. But similarity ratio fluctuated according to the impact of the noise. Compressed image can be accepted as the reliable image. The attacked images can not be approved as the authenticated image because the PSNR values are not up to the reasonable range. 


\begin{tabular}{|l|c|c|}
\hline \multirow{2}{*}{ Types of attacks } & $\begin{array}{c}\text { Detected } \\
\text { watermark } \\
\text { Quality(CPN) }\end{array}$ & $\begin{array}{c}\text { Detected } \\
\text { watermark } \\
\text { Quality (SSOM) }\end{array}$ \\
\cline { 2 - 3 } & PSNR & PSNR \\
\hline No attacks & 33 & 56.9 \\
\hline Compressed(WM) & 32.3 & 52 \\
\hline Gaussian & 32 & 50.8 \\
\hline Poisson & 32 & 51.5 \\
\hline Salt \& Pepper & 33 & 50 \\
\hline Speckle & 32 & 51 \\
\hline
\end{tabular}

Table 4.2.1. Similarity between digital watermark and detected watermark

\section{CONCLUSION}

This paper enlightens new system for watermarking. Superior_SOM (SSOM) based MSWG\&D system provides efficient watermarking technique. Previous techniques used conventional SOM as embedding vector selector. This paper actively recommends Superior_SOM for watermark value generation from the RGB colour features of host image. Since the watermark value is synthesized from the image the watermark values are highly sensitive to the image features. Hence the slight attacks will claim for reliability. By using SSOM learning efficiency tuning logics, authenticated users can maintain their watermark value identity secret. Thus this system provides one more reliable scheme for watermarking.

\section{REFERENCES}

[1] Bibi Isac and V. Santhi, "A Study on Digital Image and Video Watermarking Schemes using Neural Networks": In International Journal of Computer Applications (09758887). Volume 12-No.9. January 2011

[2] Chenthalir N, Dr. E. Ramaraj: "Magnitude of Self Systematizing Resemblance Measures in Knowledge Mining: In Software Technology and Engineering Proceedings of the International Conference on ICSTE
2009, pp.239- 3, DOI No:10.1142/97889814289986_0044, World Scientific Publications.

[3] N. Chenthalir Indra and Dr. E. Ramaraj, "Similar Dissimilar Victor Measure Analysis to Improve Image Knowledge Discovery Capacity of SOM “.: In International Conference on Information and Communication Technologies, ICT 2010 Proceedings. Volume 101, Part 2, pp.389-393, DOI: 0.1007/978-3-642-15766-0_61. published by Springer-Verlag.

[4] Chuan-Yu Chang, Sheng-Jyun Su, Hung-Jen Wang : "Using a Full Counterpropagation Neural Network for Image Watermarking", International Computer Symposium, Dec. 15- 7, 2004,pp.461-466 Taipei, Taiwan.

[5] Chuan-Yu Chang, Sheng-Jyun Su, Hung-Jen Wang : "Copyright authentication for images with a Full Counter propagation neural network", Expert Systems with applications 37, 2010.

[6] Dorina Marghescu, MikkoRajanen, Barbro Back, : "Evaluating the Quality of uses of Visual Data Mining Tools". In: Proceedings of 11th European Conference on Information Technology Evaluation, pp.239-250, (2004).

[7] Fan Zhang, Hongbin Zhang, "Applications of Neural Network to Watermarking Capacity”, International Symposium on communications and Information Technologies, October 26-29, 2004.

[8] Jun Zhang, Nenchao Wang, Feng Xiong, "Hiding a Logo Wateramrk into the Multiwavelet Domain using Neural Networks", In the Proceedings of the $14^{\text {th }}$ IEEE International Conference on Tools with Artificial Intelligence, 2002.

[9] Kumar, S., Raman, B., Thakur, M.: "Real Coded Genetic Algorithm based Stereo image Watermarking". In: IJSDIA 1(1), pp.23-33 (2009).

[10] S.S. Sujatha and M. Mohamed Sathik "Feature Based Watermarking Algorithm by Adopting Arnold Transform ICT 2010, CCIS 101, pp. 78-82, 2010. C Springer-Verlag Berlin Heidelberg 2010.

[11] S.S. Sujatha. M. Mohamed Sathik, CiiT Inernational Journal of Digital Image Processing, Vol 2, No 7, pp.185188, July 2010. 